\title{
The enantiospecific synthesis of (+)-monomorine I using a 5-endo-trig cyclisation strategy
}

Malcolm B. Berry ${ }^{1}$, Donald Craig ${ }^{* 1}$, Philip S. Jones ${ }^{2}$ and Gareth J. Rowlands ${ }^{1,3}$

\section{Full Research Paper}

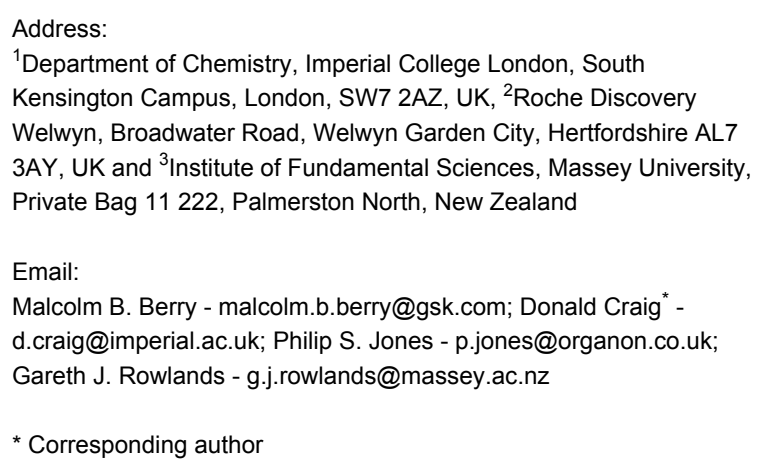

Beilstein Journal of Organic Chemistry 2007, 3, No. 39. doi:10.1186/1860-5397-3-39

Received: 03 October 2007

Accepted: 08 November 2007

Published: 08 November 2007

C 2007 Berry et al; licensee Beilstein-Institut License and terms: see end of document.

\begin{abstract}
We have developed a general strategy for the synthesis of 2,5-syn disubstituted pyrrolidines that is based on the multi-faceted reactivity of the sulfone moiety and a 5-endo-trig cyclisation. This methodology was applied to the synthesis of indolizidine alkaloid monomorine I. Two factors were key to the success of this endeavour; the first was the choice of nitrogen protecting group whilst the second was the conditions for the final stereoselective amination step. Employing a combination of different protecting groups and an intramolecular reductive amination reaction we were able to prepare (+)-monomorine I in just 11 steps from commercially available D-norleucine in a completely stereoselective manner.
\end{abstract}

\section{Background}

The abundance in natural products and drug candidates of saturated five-membered heterocycles, such as tetrahydrofurans and pyrrolidines, makes these motifs attractive targets for synthesis. Over the last decade we have developed a powerful general strategy for the preparation of such compounds based upon the multi-faceted reactivity of the sulfone group and the formally disfavoured 5-endo-trig mode of cyclisation. [1-6] The methodology allows the conversion of epoxides $(\mathrm{X}=\mathrm{O})$ or aziridines $(\mathrm{X}=\mathrm{N}-\mathrm{PG})$ (2) into the desired trisubstituted tetrahydrofurans or pyrrolidines (5) via a series of sulfone-mediated transformations (Scheme 1). Ring-opening 2 with the sulfone-stabilised anion of $\mathbf{1}$ forms the first $\mathrm{C}-\mathrm{C}$ bond and furnishes $\mathbf{3}$. Modifica-

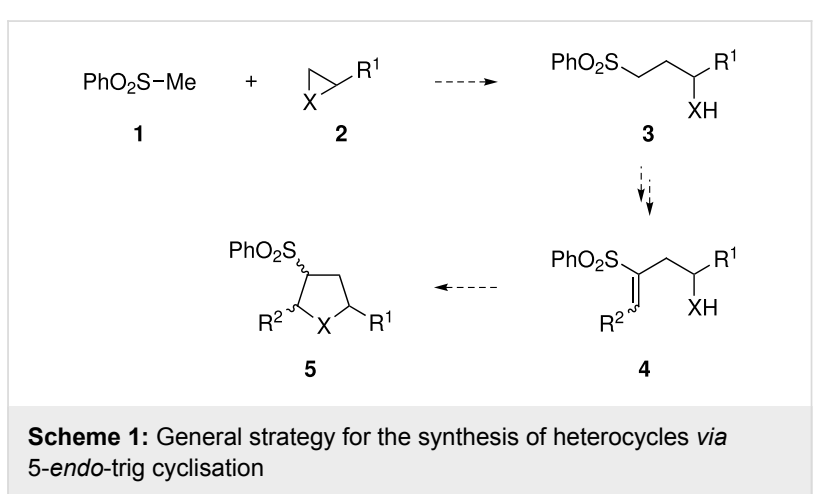


tion of the work of Julia [7-9] then utilises the sulfone to facilitate stereocontrolled alkenylation to give the cyclisation substrate 4. Finally, 5-endo-trig cyclisation yields the desired heterocycles 5. Overall, the sulfone moiety enables two C-C bond forming steps, allows stereocontrol of the alkene and activates the alkene to cyclisation. Furthermore, the sulfone can be used to elaborate the basic framework post-cyclisation.

In this publication we outline the application of this methodo$\operatorname{logy}$ to the synthesis of the indolizidine, $(+)$-monomorine I. [10-13] We have briefly described this work in a previous communication. [4]

\section{Results and Discussion}

The pyrrolidine ring is an important structural motif that occurs in a range of pheromones, venoms and drug candidates. [14] In order to demonstrate the synthetic utility of the sulfone-mediated 5-endo-trig methodology. [3] we embarked on the total synthesis of the indolizidine alkaloid monomorine I, the trail pheromone of the Pharaoh worker ant Monomorium pharaonis. [10] Our initial synthetic plan is outlined in Scheme 2; aziridine 6, prepared from D-norleucine by standard transformations, would be converted into the 2,5-syn disubstituted pyrrolidine core 8 via alkene 7 . With all the required carbon atoms in place, the final steps would involve deprotection, intramolecular hydroamination of the alkene and desulfonylation.

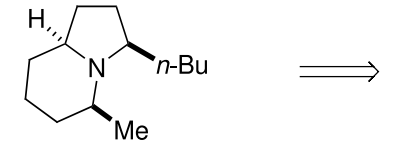

(+)-monomorine I

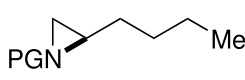

6

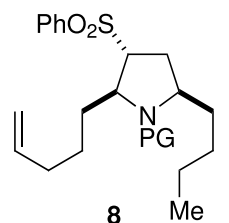

$\Downarrow$

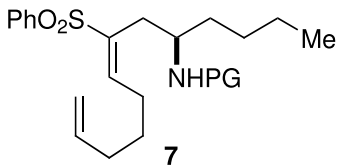

Scheme 2: Retrosynthesis of (+)-monomorine I

Initial studies directed towards this goal exploited the tosyl moiety as the nitrogen-protecting group (PG) and resulted in a succinct synthesis of alkenes of the type 4 (X = NTs; Scheme 1). [15] Disappointingly, all attempts to ring-close the sulfonamides proved fruitless, and it was found that desulfonylation was necessary before cyclisation could be achieved. Whilst the tosyl-based methodology permitted the synthesis of a range of simple, non-functionalised pyrrolidines $5(X=N H)$, the harsh nature of the deprotection reaction, treatment with hydrobromic acid and phenol in acetic acid at reflux, led to the destruction of the the terminal alkene functionality of $7(\mathrm{PG}=\mathrm{Ts}$; Scheme 2) required for our synthesis of (+)-monomorine I. As a result of this set-back, a second nitrogen protecting group was assessed. The diphenylphosphinyl group $\left(\mathrm{PG}=\mathrm{P}(\mathrm{O}) \mathrm{Ph}_{2}=\mathrm{Dpp}\right)$ overcame many of the problems encountered with the tosyl group; protected alkenes $4(\mathrm{X}=\mathrm{NDpp})$ underwent smooth 5-endo-trig cyclisation to furnish $N$-(diphenylphosphinyl)pyrrolidines 5 (X $=$ Dpp) in good yields. $[3,16]$ Furthermore, dephosphinylation was readily achieved under either Lewis acidic or Brønsted acid conditions compatible with a range of functional groups. This second-generation methodology was limited by the finding that acylation of $\mathbf{3}(\mathrm{X}=\mathrm{NDpp})$ could only be achieved with non-enolisable acid chlorides, rendering it unsuitable for the synthesis of $(+)$-monomorine I. Ultimately, no single protecting group was found to be suitable and it was necessary to exploit a combination of protecting groups. The full evolution of the 5-endo-trig cyclisation-based pyrrolidine methodology will be described in a future publication.

Key to the successful synthesis of (+)-monomorine I was the use of the $N$-(benzoyl)aminosulfone 11 (Scheme 3). Benzamide 11 could be prepared from $N$-(diphenylphosphinyl)aziridine 9 by ring-opening with 1 followed by protecting group interchange. Although this strategy was not as elegant as utilising an $N$-benzoylaziridine directly, we deemed it prudent not to

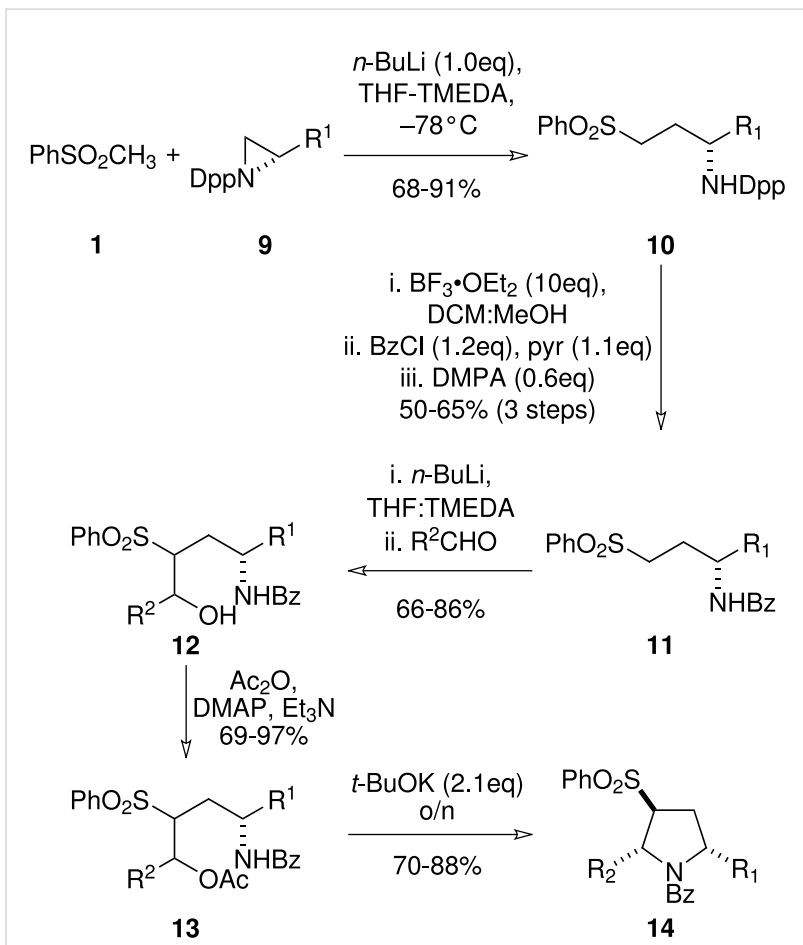

Scheme 3: The sulfone-mediated synthesis of 2,5-syn disubstituted pyrrolidines via a 5-endo-trig cyclisation 


\begin{tabular}{|c|c|c|c|c|c|c|}
\hline Pyrrolidine & $\mathbf{R}^{1}$ & $\mathbf{R}^{2}$ & Reagent & $\mathbf{R}^{3}$ & Product & Yield (\%) \\
\hline $14 a$ & $i \mathrm{Pr}$ & $\mathrm{C}_{6} \mathrm{H}_{13}$ & $\mathrm{HCl}$ & $\mathrm{H}$ & $15 a$ & 69 \\
\hline $14 b$ & $\mathrm{CH}_{2} \mathrm{iPr}$ & $\mathrm{C}_{6} \mathrm{H}_{13}$ & $\mathrm{HCl}$ & $\mathrm{H}$ & $15 b$ & 60 \\
\hline $14 a$ & $i \mathrm{Pr}$ & $\mathrm{C}_{6} \mathrm{H}_{13}$ & Super-Hydride ${ }^{\circledR}$ & $\mathrm{H}$ & $15 a$ & 69 \\
\hline $14 c$ & $i \operatorname{Pr}$ & $\left(\mathrm{CH}_{2}\right)_{3} \mathrm{CH}=\mathrm{CH}_{2}$ & Super-Hydride ${ }^{\circledR}$ & $\mathrm{H}$ & $15 c$ & 57 \\
\hline $14 c$ & $i \mathrm{Pr}$ & $\left(\mathrm{CH}_{2}\right)_{3} \mathrm{CH}=\mathrm{CH}_{2}$ & DIBAL & $\mathrm{Bn}$ & $16 c$ & 70 \\
\hline $14 d$ & $\mathrm{Bn}$ & $\mathrm{Me}$ & DIBAL & $\mathrm{Bn}$ & $16 d$ & 67 \\
\hline
\end{tabular}

subject such a species to nucleophilic attack due to reported issues with chemoselectivity. [17] Careful optimisation obviated the need for chromatography following the protecting group exchange, and the benzamides $\mathbf{1 1}$ could be isolated in high purity and good yield. Hydroxyalkylation with a range of aldehydes proceeded without issue to give the $\beta$-hydroxysulfones 12 in excellent yields. The $\beta$-hydroxysulfones were then acylated under standard conditions to give $\mathbf{1 3}$. Treatment of the $\beta$-acetoxysulfones $\mathbf{1 3}$ with two equivalents of base gave the pyrrolidines $\mathbf{1 4}$ directly as the product of a one-pot eliminationcyclisation cascade. The pyrrolidines were formed with complete diastereoselectivity for the 2,5-syn diastereoisomers. Although this stereochemical relationship could not be

14a; $\mathrm{R}^{1}=\mathbb{P r} ; \mathrm{R}^{2}=\mathrm{C}_{6} \mathrm{H}_{13}$
$14 \mathbf{b} ; \mathrm{R}^{1}=\mathrm{CH}_{2} \mathrm{Pr} ; \mathrm{R}^{2}=\mathrm{C}_{6} \mathrm{H}_{13}$
$14 \mathbf{c} ; \mathrm{R}^{1}=\mathrm{Pr} ; \mathrm{R}^{2}=\left(\mathrm{CH}_{2}\right)_{3} \mathrm{CH}=\mathrm{CH}_{2}$
14d; $\mathrm{R}^{1}=\mathrm{Bn} ; \mathrm{R}^{2}=\mathrm{Me}$
Scheme 4: The deprotection of $\mathrm{N}$-benzoylpyrrolidines

discerned from the ${ }^{1} \mathrm{H}$ NMR spectra of $\mathbf{1 4}$ due to peak broadening caused by amide rotamers, a combination of further elaboration and X-ray crystallographic analysis confirmed the assignment.

Deprotection of simple benzoyl-protected pyrrolidines 14a and 14b could be achieved by acid hydrolysis (Scheme 4 and Table 1). However, as with the tosyl-based methodology, such reaction conditions were incompatible with the terminal alkenesubstituted pyrrolidine 14c. Therefore alternative deprotection conditions were investigated. Attempted base-mediated hydrolysis led to formation of the $N$-benzoylaminosulfone 11, presumably by a sequence involving ring-opening by elimination, hydration of the electron-deficient alkenyl sulfone double bond and retro-aldol-like fragmentation. Reductive deprotection proved to be a more fruitful avenue of study. After considerable optimisation it was found that treatment of the $N$-benzoylpyrrolidines with Super-Hydride ${ }^{\circledR}[18]$ gave the free amines 15, whilst the use of DIBAL in THF furnished the benzyl-protected pyrrolidines $\mathbf{1 6}$ in good yield (Scheme 4 and Table 1).

With the chemistry now in place to undertake the synthesis of (+)-monomorine I, the initial target, pyrrolidine 22, was

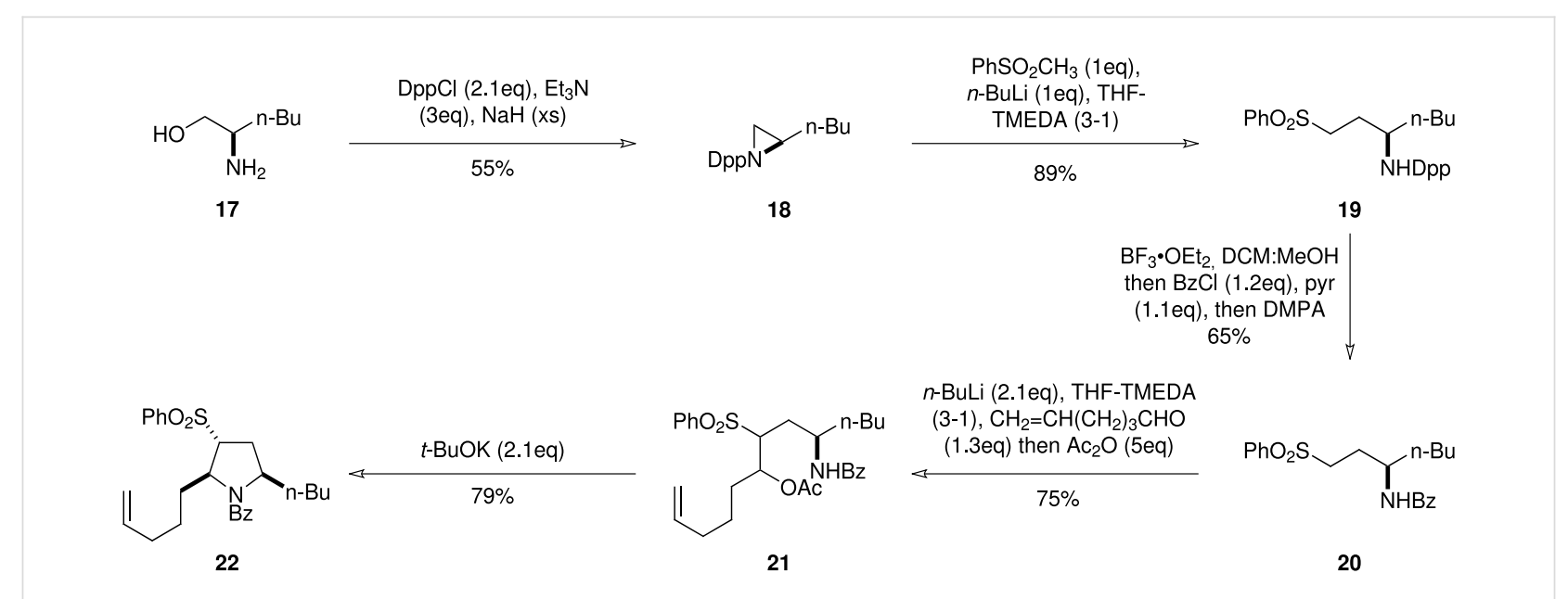

Scheme 5: Synthesis of $\mathrm{N}$-benzoyl protected pyrrolidine required for the preparation of (+)-monomorine I 


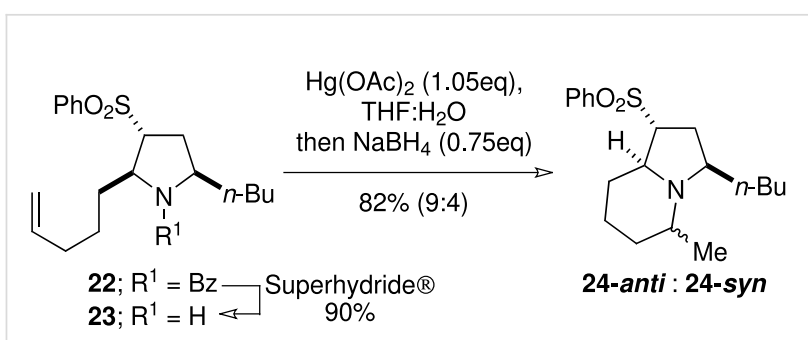

Scheme 6: Mercury-mediated hydroamination

prepared. Commercially available D-norleucine was reduced to the amino alcohol 17. [19] This was then converted into the benzoyl-protected aminosulfone 20 via the diphenylphosphinylaziridine 18, which was ring-opened to give 19 , followed by protecting group exchange (Scheme 5). Formation of the dianion of $\mathbf{2 0}$ by exposure to two equivalents of $n$-butyllithium, followed by reaction with hex-5-enal and in situ trapping of the intermediate alkoxides gave the ester $\mathbf{2 1}$ as predominantly one diastereoisomer. Finally, one-pot elimination-cyclisation, promoted by two equivalents of potassium tert-butoxide, furnished the 2,5-syn-pyrrolidine $\mathbf{2 2}$ as a single diastereoisomer. Concurrently with the synthesis of 22, the isopropyl model system, $\mathbf{1 4 c}$, was prepared using analogous chemistry.

Deprotection of $\mathbf{2 2}$ and $\mathbf{1 4 c}$ was readily achieved with SuperHydride ${ }^{\circledR}$ to give the free amines 23 and $\mathbf{1 5 c}$, which were subjected to mercury-mediated hydroamination (Scheme 6 and Scheme 7). [20] Cyclisation of $\mathbf{2 3}$ proceeded in good yield to give a 9:4 mixture of two indolizidines, epimeric at the C-5 methyl group 24-anti and 24-syn (Scheme 6). Cyclisation of the isopropyl analogue $\mathbf{1 5}$ proceeded with improved stereoselectivity to give a 5:1 mixture of epimeric indolizidines 26-anti

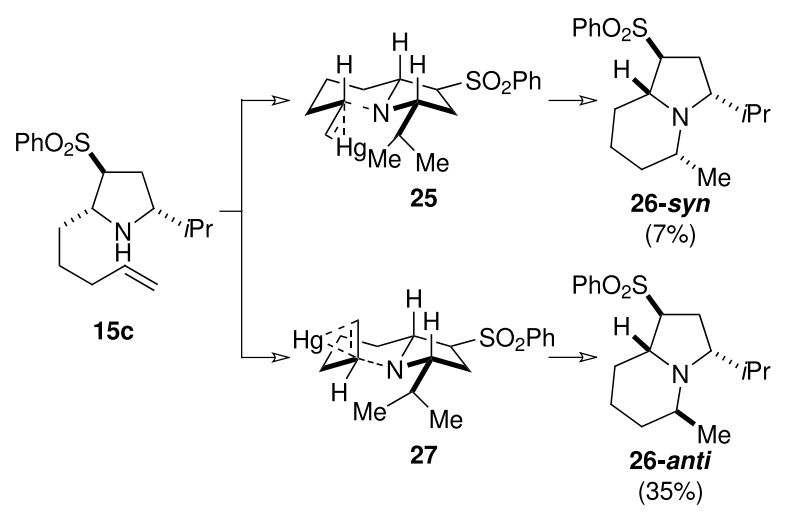

Scheme 7: Proposed transition state for mercury-mediate hydroamination

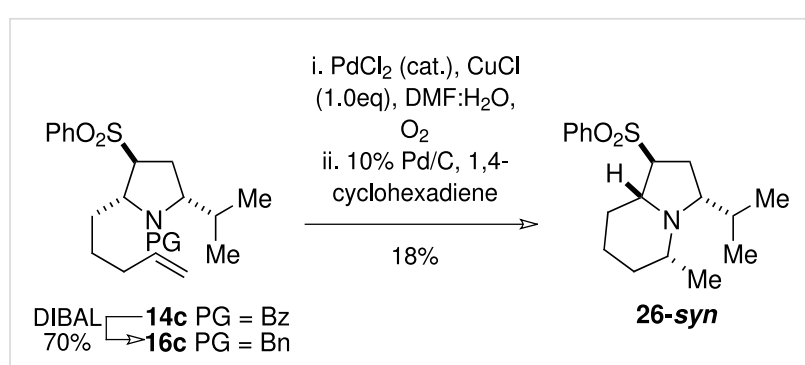

Scheme 8: Model study for the reductive amination-based cyclisation

and 26-syn (Scheme 7). Presumably, the increased steric bulk of the isopropyl group is responsible for the higher antiselectivity. Assignment of the relative stereochemistry of the epimeric pairs proved problematic due to difficulties encountered during separation, and the presence of overlapping signals in the ${ }^{1} \mathrm{H}$ NMR spectrum. Finally, a combination of X-ray diffraction analysis and comparison of the ${ }^{1} \mathrm{H}$ NMR showed that the major diastereoisomer in each case was the undesired C-5 epimer, with the methyl group residing in the axial position. Naturally, we had assumed that the diastereoisomer in which all the substituents adopted a pseudo-equatorial orientation would have been formed preferentially. Yet inspection of the possible transition states for the cyclisation 25 vs. 27 reveals that the axial methyl may be favoured so as to minimize the strain associated with the eclipse of the C-3 and C-5 substituents (Scheme 7). Branching of the isopropyl substituent would cause greater interaction than the butyl group, and therefore would lead to an increase in selectivity.

The findings described above dictated that an alternative cyclisation strategy be investigated. It was anticipated that intramolecular reductive amination of a pendant methyl ketone would furnish the correct diastereoisomer, because the hydride source would be expected to approach the iminium ion from the less sterically demanding face, with the C-9 stereocentre being the controlling factor. [21] Both the benzoyl protecting group and the free amine were deemed incompatible with such a strategy. Therefore, 22 and $\mathbf{1 4 c}$ were converted into the benzyl-protected pyrrolidines $\mathbf{2 8}$ and $\mathbf{1 6 c}$ respectively by partial reduction with DIBAL-H (s Scheme 8 and Scheme 9). Wacker oxidation[22] of the isopropyl model compound 16c gave the desired methyl ketone, which was subjected to transfer hydrogenation. [23] The latter reaction precipitated a reaction cascade commencing with deprotection of the $\mathrm{N}$-benzylpyrrolidine followed by intramolecular reductive amination to give the desired indolizidine 26-syn as a single diastereoisomer in $18 \%$ yield for the two steps. Whilst the yield of this unoptimised reaction was not satisfactory, we were pleased to observe that only the desired diastereoisomer was formed. 


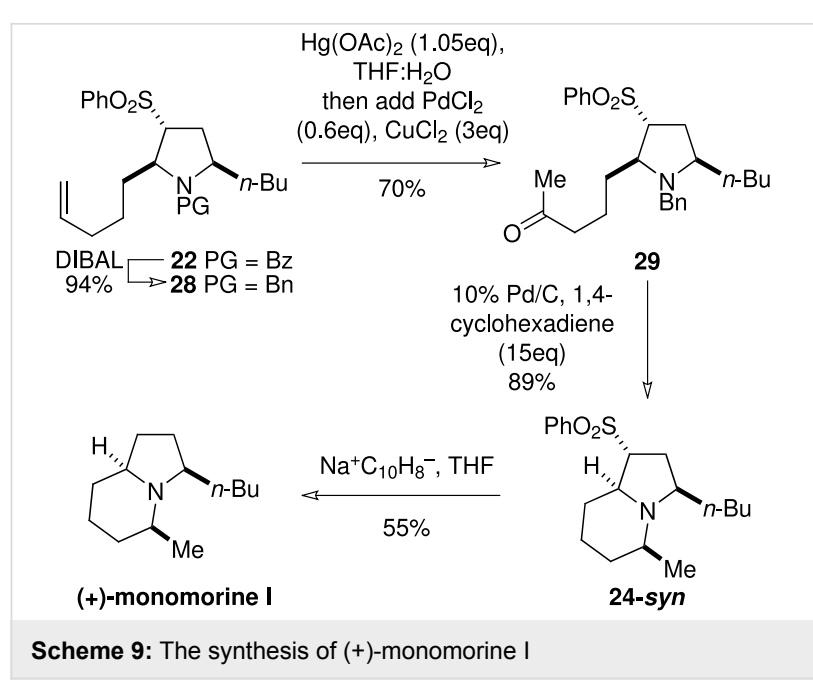

Oxidation of the terminal alkene of $\mathbf{2 8}$ under Wacker conditions proved highly capricious and was ultimately abandoned in favour of a more reliable oxymercuration protocol. [24] Under these conditions the methyl ketone $\mathbf{2 9}$ was isolated in $70 \%$ yield (Scheme 9). Catalytic transfer hydrogenation led to sequential debenzylation and intramolecular reductive amination to furnish 24-syn as a single diastereoisomer in excellent yield. Desulfonylation was achieved by brief exposure of 24-syn to sodium naphthalenide in THF to furnish (+)monomorine I, which showed ${ }^{1} \mathrm{H}$ and ${ }^{13} \mathrm{C}$ NMR, IR, mass spectral and optical rotation characteristics in agreement with published values. [25] Short reaction times were found to be crucial to the success of this reaction.

In summary, we have developed a highly stereoselective 5 -endo-trig cyclisation reaction that facilitates the preparation of 2,5-syn disubstituted pyrrolidines. We have used this transformation as the key step in the synthesis of the indolizidine alkaloid, (+)-monomorine I. The synthesis was achieved in nine steps from the readily available aziridine $\mathbf{1 8}$, and compares favourably with other total syntheses in the literature.

See Supporting Information File 1 for full experimental data.

\section{Supporting Information}

\section{Supporting Information File 1}

The enantiospecific synthesis of $(+)$-monomorine I using a 5-endo-trig cyclisation strategy: full experimental data. Full preparative details of all compounds prepared are reported, together with their spectroscopic data. [http://www.beilstein-journals.org/bjoc/content/ supplementary/1860-5397-3-39-S1.doc]

\section{References}

1. Caldwell, J. J.; Craig, D.; East, S. P. Synlett 2001, 1602-1604. doi:10.1055/s-2001-17440

2. Craig, D.; Ikin, N. J.; Mathews, N.; Smith, A. M. Tetrahedron 1999, 55, 13471-13494. doi:10.1016/S0040-4020(99)00832-7

3. Craig, D.; Jones, P. S.; Rowlands, G. J. Synlett 1997, 1423-1425. doi:10.1055/s-1997-1054

4. Berry, M. B.; Craig, D.; Jones, P. S.; Rowlands, G. J. Chem. Commun. 1997, 2141-2142. doi:10.1039/a706333d

5. Craig, D.; Ikin, N. J.; Mathews, N.; Smith, A. M. Tetrahedron Lett. 1995, 36, 7531-7534. doi:10.1016/0040-4039(95)01524-8

6. Craig, D.; Smith, A. M. Tetrahedron Lett. 1992, 33, 695-698. doi:10.1016/S0040-4039(00)92345-1

7. Cuvigny, T.; Dupenhoat, C. H.; Julia, M. Tetrahedron 1986, 42, 5329-5336. doi:10.1016/S0040-4020(01)82082-2

8. Julia, M.; Stacino, J. P. Tetrahedron 1986, 42, 2469-2474. doi:10.1016/0040-4020(86)80010-2

9. Julia, M.; Launay, M.; Stacino, J. P.; Verpeaux, J. N. Tetrahedron Lett. 1982, 23, 2465-2468. doi:10.1016/S0040-4039(00)87369-4

10. Ritter, F. J.; Rotgans, I. E. M.; Talman, E.; Verwiel, P. E. J.; Stein, F. Experientia 1973, 29, 530-531. doi:10.1007/BF01926645

11. Conchon, E.; Gelas-Mialhe, Y.; Remuson, R. Tetrahedron: Asymmetry 2006, 17, 1253-1257. doi:10.1016/j.tetasy.2006.04.027

12. Amos, R. I. J.; Gourlay, B. S.; Molesworth, P. P.; Smith, J. A.; Sprod, O. R. Tetrahedron 2005, 61, 8226-8230. doi:10.1016/j.tet.2005.06.026

13. Amat, M.; Llor, N.; Hidalgo, J.; Escolano, C.; Bosch, J. J. Org. Chem. 2003, 68, 1919-1928. doi:10.1021/jo0266083

14. Michael, J. P. Nat. Prod. Rep. 2005, 22, 603-626. doi:10.1039/ b413748p

15. Berry, M. B. Ph.D. Thesis, University of London, Department of Chemistry, Imperial College: London, UK, 1993.

16. Rowlands, G. J. Ph.D. Thesis, University of London, Department of Chemistry, Imperial College: London, UK, 1996.

17. Baldwin, J. E.; Adlington, R. M.; Robinson, N. G. J. Chem. Soc., Chem. Commun. 1987, 153-155. doi:10.1039/c39870000153

18. Brown, H. C.; Kim, S. C. Synthesis 1977, 635-636. doi:10.1055/s1977-24511

19. McKennon, M. J.; Meyers, A. I.; Drauz, K.; Schwarm, M. J. Org. Chem. 1993, 58, 3568-3571. doi:10.1021/jo00065a020

20. Perie, J. J.; Lattes, A.; Laval, J. P.; Roussel, J. Tetrahedron 1972, 28 , 675. doi:10.1016/0040-4020(72)84031-6

21. Stevens, R. V.; Lee, A. W. M. J. Chem. Soc., Chem. Commun. 1982, 102-103. doi:10.1039/c39820000102

22. Tsuji, J. Synthesis 1984, 369-384. doi:10.1055/s-1984-30848

23. Felix, A. M.; Heimer, E. P.; Lambros, T. J.; Tzougraki, C.; Meienhofer, J. J. Org. Chem. 1978, 43, 4194-4196. doi:10.1021/jo00415a045

24. Rodeheaver, G. T.; Hunt, D. F. J. Chem. Soc. D 1971, 818. doi:10.1039/c29710000818

25. Munchhof, M. J.; Meyers, A. I. J. Am. Chem. Soc. 1995, 117, 5399-5400. doi:10.1021/ja00124a039 


\section{License and Terms}

This is an Open Access article under the terms of the Creative Commons Attribution License

(http://creativecommons.org/licenses/by/2.0), which permits unrestricted use, distribution, and reproduction in any medium, provided the original work is properly cited.

The license is subject to the Beilstein Journal of Organic Chemistry terms and conditions:

(http://www.beilstein-journals.org/bjoc)

The definitive version of this article is the electronic one which can be found at:

doi:10.1186/1860-5397-3-39 